\title{
Control properties of multiagent dynamical systems modelling brain neural networks
}

\author{
M.Isabel Garcia-Planas \\ Department de Matemàtiques \\ Universitat Politècnica de Catalunya \\ Barcelona, Spain \\ maria.isabel.garcia@upc.edu
}

\begin{abstract}
The control of linear dynamical systems is a strategy that the brain uses to control its own intrinsic dynamics. The brain structure can be modelled as a networked system that is expressly interesting system to control because of the role of the underlying architecture, which predisposes some components to particular control motions. The concept of brain cognitive control defined by neuroscientists is related to the mathematical concept of control defined by physicists, mathematicians, and engineers, where the state of a complex system can be adjusted by a particular input. The in-depth study on the controllability and structural controllability character of linear dynamical systems, despite being very difficult, could help to regulate the brain cognitive function. Small advances in the study can favour the study and action against learning difficulties such as dyscalculia or other disturbances like the phenomena of forgetting. Between different aspects in which we can study the controllability we have the notion of structural controllability and exact controllability. In this talk, we revise these concepts for linear dynamical systems and multiagent neural networks.

Index Terms-neural network, controllability, exact controllability, eigenvalues, eigenvectors, linear systems
\end{abstract}

\section{INTRODUCTION}

Brain networks have become a productive field of research so-called or network neuroscience, covering across different areas, from interacting biomolecules all the way to social behavior. A significant driving force has been the use of mathematical tools to neurobiological systems, especially models and measures of graph theory and dynamical networks systems theory [21].

A complex dynamical network consists of a large set of interconnected nodes, with each node being a fundamental unit with detailed contents. More specifically, an artificial neural network (ANN) is a computational model that is loosely inspired by the human brain consisting of an interconnected network of simple processing units that can learn from experience by modifying its connections.

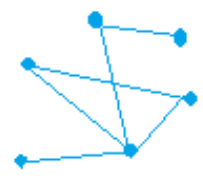

Fig. 1. Neural Network.
The neuronal network locution refers to a particular mathematical model for understanding brain function, in which neurons are the basic computational units, and the calculation interprets network interactions.

The mathematical models of neural networks, as Kriegeskorte says in [14], are the starting point of a new vision of neuroscience, the so-called computational neuroscience, which allows us to deal with real-world tasks that require extensive knowledge and complex computations.

Neural systems allow humans to make multiple complex mental processes that permit to make any task necessary for daily life. This kind of systems has the ability to alter their dynamics to meet the demands of the subject in the processes of reception, selection, transformation, storage, processing and retrieval of information. This ability is called control of neural systems.

Brain cognitive control is one of several classes of cognitive processes that are essential to good human functioning. This concept, understood as that capacity that regulates our behavior and that allows us to select the information that is necessary for our objective and inhibit irrelevant information is analogous to the mathematical concept of control of the dynamic systems used in engineering, where the state of a complex system can be modulated by the input of energy.

Neural network systems, such as the brain, are very attractive systems for the study of control due to their structure that predisposes certain components to specific control actions. The neuronal sets of the brain can be interpreted as the nodes of a complex system and the anatomical cables of interconnection as the axes, this system exerts an impact on the neural function. It is therefore plausible that the brain regulates cognitive function through a process of transient network level control similar to technological systems modelled mathematically as complex systems. Although the complete understanding of the relationship between mathematical control measures and the notions of cognitive control of neuroscience are difficult to achieve, small advances in the study can favour the study and action against learning difficulties such as dyscalculia or other disturbances like the phenomena of forgetting, ( [9], [10]).

In these recent years, the study of the control of complex networks with linear dynamics has gained importance in both science and engineering. Controllability of a dynamical system has being largely studied by several authors and under many 
different points of view, (see [1], [2], [3], [5], [8], [12], [15] and [20], for example). Between different aspects in which we can study the controllability we have the notion of structural controllability that has been proposed by Lin [18] as a framework for studying the controllability properties of directed complex networks where the dynamics of the system is governed by a linear system: $\dot{x}(t)=A x(t)+B u(t)$ usually the matrix $A$ of the system is linked to the adjacency matrix of the network, $x(t)$ is a time dependent vector of the state variables of the nodes, $u(t)$ is the vector of input signals, and $B$ which defines how the input signals are connected to the nodes of the network and it is the called input matrix. Structurally controllable means that there exists a matrix $\bar{A}$ in which is not allowed to contain a non-zero entry when the corresponding entry in A is zero such that the network can be driven from any initial state to any final state by appropriately choosing the input signals $u(t)$. Recent studies over the structural controllability can be found on [19].

Another important aspect of control is the notion exact controllability concept following definition given in [7], [24]. This concept is based on the maximum multiplicity to identify the minimum set of driver nodes required to achieve full control of networks with arbitrary structures and link-weight distributions.

In this paper we revise these concepts for multiagent neural network and it is structured as follows. The main definitions and results about graph theory and control concepts of linear systems used throughout the paper are presented in a preliminary section. In section III, the structural and exact control concepts are generalized to multiagent systems with a topology connecting the agents. Finally, some conclusions are presented.

\section{Preliminaries}

\section{A. Graphs}

Mathematical networks are usually referred to as graphs, and the field concerning its study is called graph theory.

Roughly speaking, a graph is a set of objects (called vertices or nodes) that are connected together. The connections between the vertices are called edges.

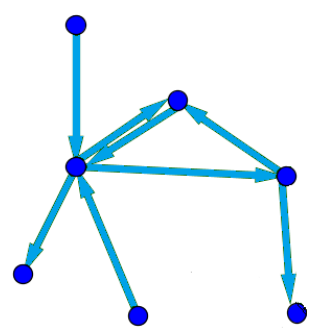

Fig. 2. A directed graph with 7 vertices and 8 edges.

We consider a graph $\mathcal{G}=(\mathcal{V}, \mathcal{E})$ of order $n$ with the set of vertices $\mathcal{V}=\{1, \ldots, n\}$ and the set of edges $\mathcal{E}=\{(i, j) \mid$ $i, j \in \mathcal{V}\} \subset \mathcal{V} \times \mathcal{V}$

Given an edge $(i, j) i$ is called the parent node and $j$ is called the child node and $j$ is in the neighbor of $i$, concretely we define the neighbor of $i$ and we denote it by $\mathcal{N}_{i}$ to the set $\mathcal{N}_{i}=\{j \in \mathcal{V} \mid(i, j) \in \mathcal{E}\}$. In the case where $j=i$ the edge is called self-loop

The graph is called undirected if verifies that $(i, j) \in \mathcal{E}$ if and only if $(j, i) \in \mathcal{E}$. The graph is called connected if there exists a path between any two vertices, otherwise is called disconnected.

Associated to the graph we consider a matrix $A=\left(a_{i j}\right)$ (notation: $i$-row, $j$-column) called (unweighted) adjacency matrix defined as follows, $a_{i j}=1$ if $(i, j) \in \mathcal{E}$, and $a_{i j}=0$ otherwise.

In a more general case we can consider a weighted adjacency matrix is $A=\left(a_{i j}\right)$ with $a_{i j} \neq 0$ if $(i, j) \in \mathcal{E}$, and $a_{i j}=0$ otherwise, in this case the graph is called weighted graph.

Notice that, if self-loops are not allowed $a_{i i}=0$.

The graph is called simple if it is unweighted, undirected and containing no self-loops.

1) Graph Laplacian: The Laplacian matrix of the simple graph is

$$
\mathcal{L}=\left(l_{i j}\right)= \begin{cases}\left|\mathcal{N}_{i}\right| & \text { if } i=j \\ -1 & \text { if } j \in \mathcal{N}_{i} \\ 0 & \text { otherwise }\end{cases}
$$

The Laplacian matrix $\mathcal{L}$ of a simple graph is a positive semi-definite operator.

Given an undirected weighted graph its Laplacian matrix $\mathcal{L}$ is the square matrix of size $n$ defined by $\mathcal{L}=D-W$; where $W$ is the weighted adjacency matrix representing the weights of the edge set $\mathcal{E}$ i.e $W=\left(a_{i j}\right)$, and where $D=$ $\operatorname{diag}\left(D_{1}, \ldots, D_{n}\right)$ with $D_{j}=\sum_{i=1}^{n} a_{i j}$, for $1 \leq j \leq n$,

$D_{1}$ is the sum all weights of the edges arriving to the node 1.

Notice that if the graph is simple, i.e. the non-zero weights are 1 and no contains self-loops, this definition coincides with the previous one. For a general graph

Example 1: Let us consider the following deep recurrent neural network (3)

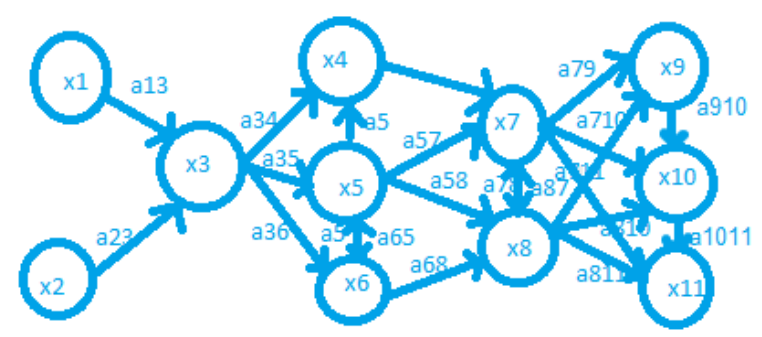

Fig. 3. Neural Network.

The adjacency matrix corresponding to this graph is as follows ( [6]) 


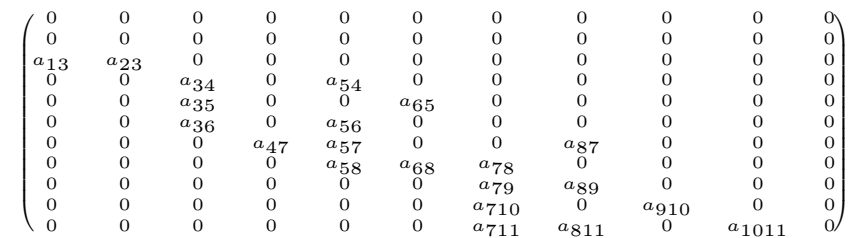

and the Laplacian matrix is

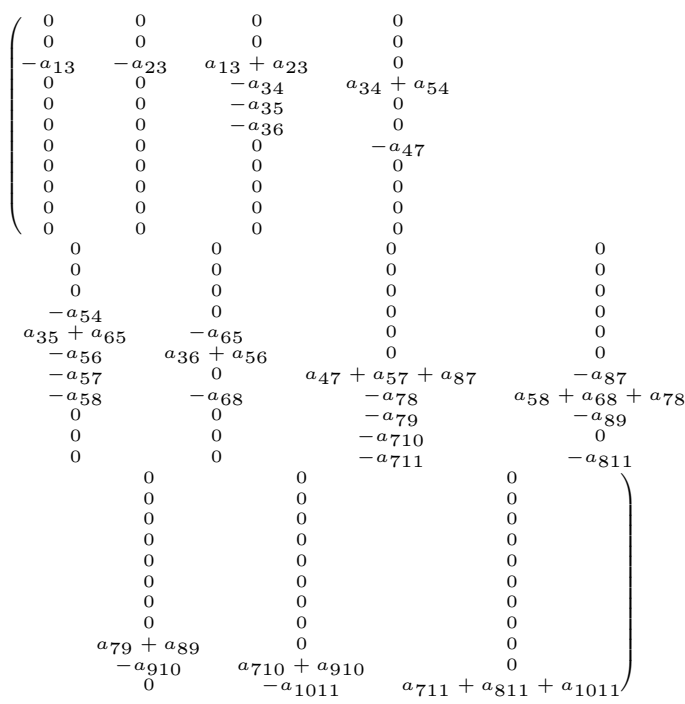

For more details about graph theory see [23].

A possible way to study the control of neural networks can be by associating a dynamic system, in the form:

$$
\dot{x}(t)=A x(t)+B u(t)
$$

where

$x=\left(\begin{array}{lll}x_{1} & \ldots & x_{n}\end{array}\right)^{t}$ stands for the states nodes, $A=\left(a_{i j}\right)$ is the adjacency matrix to a subgraph where $a_{i j}$ represents the weight of a directed link from node $i$ to $j, u$ is the vector of $m$ controllers: $u=\left(u_{1}, \ldots, u_{m}\right)^{t}$ and $B$ is the $n \times m$ control matrix.

Example 2:

Let us consider the following graph 4

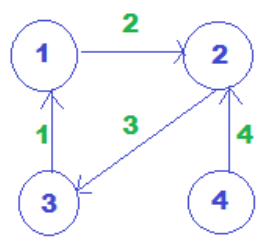

Fig. 4. Graph.

The matrices $A$ and $B$ defining the system $\dot{x}(t)=A x(t)+$ $B u(t)$ associated to this graph are

$$
A=\left(\begin{array}{ccc}
0 & 0 & a_{1} \\
a_{2} & 0 & 0 \\
0 & a_{3} & 0
\end{array}\right), B=\left(\begin{array}{c}
0 \\
a_{4} \\
0
\end{array}\right) .
$$

The subgraph corresponding to the matrix $A$ is:

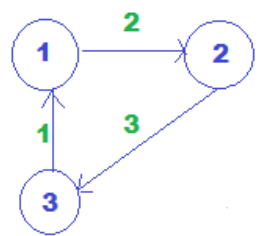

Fig. 5. Graph.

Example 3: Let us consider the graph corresponding to the recurrent neural network in figure (3). It is possible to associate it a linear control system $\dot{x}(t)=A x(t)+B u(t)$ with

$$
A=\left(\begin{array}{ccccccccc}
0 & 0 & 0 & 0 & 0 & 0 & 0 & 0 & 0 \\
a_{34} & 0 & a_{54} & 0 & 0 & 0 & 0 & 0 & 0 \\
a_{35} & 0 & 0 & a_{65} & 0 & 0 & 0 & 0 & 0 \\
a_{36} & 0 & a_{56} & 0 & 0 & 0 & 0 & 0 & 0 \\
0 & a_{47} & a_{57} & 0 & 0 & a_{87} & 0 & 0 & 0 \\
0 & 0 & a_{58} & a_{68} & a_{78} & 0 & 0 & 0 & 0 \\
0 & 0 & 0 & 0 & a_{79} & a_{89} & 0 & 0 & 0 \\
0 & 0 & 0 & 0 & a_{710} & 0 & a_{910} & 0 & 0 \\
0 & 0 & 0 & 0 & a_{711} & a_{811} & 0 & a_{1011} & 0
\end{array}\right),
$$

and

$$
B=\left(\begin{array}{cc}
a_{13} & a_{23} \\
0 & 0 \\
0 & 0 \\
0 & 0 \\
0 & 0 \\
0 & 0 \\
0 & 0 \\
0 & 0 \\
0 & 0
\end{array}\right)
$$

Notice that the graph corresponding to the system $\dot{x}(t)=$ $A x(t)+B u(t)$ there is no arc pointing toward any of the $m$ vertices with labels $n+1$ to $n+m$, since the matrix $\left(\begin{array}{ll}A & B\end{array}\right)$ only has $n$ rows.

2) Matrix Kronecker product: Remember that $A=\left(a_{i j}\right) \in$ $M_{n \times m}(\mathbb{C})$ and $B=\left(b_{i j}\right) \in M_{p \times q}(\mathbb{C})$ the Kronecker product (see [16] for more information) is defined as follows

\section{Definition 4:}

Let $A=\left(a_{j}^{i}\right) \in M_{n \times m}(\mathbb{C})$ and $B \in M_{p \times q}(\mathbb{C})$ be two matrices, the Kronecker product of $A$ and $B$, write $A \otimes B$, is the matrix

$$
A \otimes B=\left(\begin{array}{cccc}
a_{1}^{1} B & a_{2}^{1} B & \ldots & a_{m}^{1} B \\
a_{1}^{2} B & a_{2}^{2} B & \ldots & a_{m}^{2} B \\
\vdots & \vdots & & \vdots \\
a_{1}^{n} B & a_{2}^{n} B & \ldots & a_{m}^{n} B
\end{array}\right) \in M_{n p \times m q}(\mathbb{C})
$$

Kronecker product verifies the following properties

1) $(A+B) \otimes C=(A \otimes C)+(B \otimes C)$

2) $A \otimes(B+C)=(A \otimes B)+(A \otimes C)$

3) $(A \otimes B) \otimes C=A \otimes(B \otimes C)$

4) $(A \otimes B)^{t}=A^{t} \otimes B^{t}$

5) If $A \in G l(n ; \mathbb{C})$ and $B \in G l(p ; \mathbb{C}))$, then $A \otimes B \in$ $G l(n p ; \mathbb{C}))$ and $(A \otimes B)^{-1}=$ $A^{-1} \otimes B^{-1}$

6) $(A \otimes B)(C \otimes D)=(A C) \otimes(B D)$

Associated to the Kronecker product, can be defined the vectorizing operator that transforms any matrix $A$ into a column vector, by placing the columns in the matrix one after another, 


\section{Definition 5:}

Let $X=\left(x_{j}^{i}\right) \in M_{n \times m}(\mathbb{C})$ be a matrix, and we denote $x_{i}=\left(x_{i}^{1}, \ldots, x_{i}^{n}\right)^{t}$ for $1 \leq i \leq m$ the $i$-th column of the matrix $X$. We define the vectorizing operator vec, as

$$
\begin{aligned}
& \text { vec }: M_{n \times m}(\mathbb{C}) \longrightarrow M_{n m \times 1}(\mathbb{C}) \\
& X \longrightarrow\left(\begin{array}{c}
x_{1} \\
x_{2} \\
\vdots \\
x_{m}
\end{array}\right)
\end{aligned}
$$

Obviously, vec is an isomorphism.

\section{B. Control concepts for dynamical systems}

1) Controllability: Controllability is one of the most important properties of dynamical systems. A system is controllable if we can drive the state variables from any initial to any desired values within a finite period of time with properly selected inputs, more concretely:

Definition 6: The system 1 is called controllable if, for any $t_{1}>0, x(0) \in \mathbb{C}^{n}$ and $w \in \mathbb{C}^{n}$, there exists a control input $u(t)$ sufficiently smooth such that $x\left(t_{1}\right)=w$.

The controllability character can be computed by means of the well-known Kalman's rank condition

The system 1 is controllable if and only if:

Proposition 7 ( [13]):

$$
\operatorname{rank}\left(\begin{array}{llll}
B & A B & \ldots & A^{n-1} B
\end{array}\right)=n
$$

or by means the Hautus Test for controllability of linear dynamical systems.

Proposition 8 ([11]):

$$
\operatorname{rank}(s I-A \quad B)=n, \forall s \in \mathbb{C} .
$$

To ensure controllability with a minimal number of inputs the brute force approach should generate $2^{N}-1$ configurations of the $B$ matrix [17]. To solve this challenging task, Y. Y. Liu et al. proposed the maximum matching algorithm based on the network representation of the A matrix to select the control1 and observer 2 nodes that ensure controllable and observable systems.

2) Structural controllability: We recall now the concept of structural controllability [18]. Structural controllability is a generalization of the controllability concept. It is of great interest because many times we know the entries of the matrices only approximately. Roughly speaking, a linear system is said to be structurally controllable if one can find a set of values for the parameters in the matrices such that the corresponding system is controllable. More concretely, the definition is as follows.

Definition 9: The linear system 1 is structurally controllable if and only if $\forall \varepsilon>0$, there exists a completely controllable linear system $\dot{x}(t)=\bar{A} x(t)+\bar{B} u(t)$, of the same structure as $\dot{x}(t)=A x(t)+B u(t)$ such that $\|\bar{A}-A\|<\varepsilon$ and $\|\bar{B}-B\|<$ $\varepsilon$.

Recall that, a linear dynamic system $\dot{x}(t)=A x(t)+B u(t)$ has the same structure as another linear dynamical system $\dot{x}(t)=\bar{A} x(t)+\bar{B} u(t)$, of the same dimensions, if for every fixed zero entry of the pair of matrices $(A, B)$, the corresponding entry of the pair of matrices $(\bar{A}, \bar{B})$ is fixed zero and vice versa.

In the case where $B \in M_{n \times 1}$ we have that if $\dot{x}(t)=$ $A x(t)+B u(t)$ is structurally controllable, then almost every real $\dot{x}(t)=\bar{A} x(t)+\bar{B} u(t)$ with the same pattern of zero entries as $\dot{x}(t)=A x(t)+B u(t)$ will be controllable. It suffices to observe that changing the non-zero entries in the system $\dot{x}(t)=A x(t)+B u(t)$ for parameters (one parameter for each entry) we obtain a family of systems $\dot{x}(t)=A(\lambda) x(t)+B(\lambda) u(t)$ of which the non-controllable ones are the systems whose parameters are solution of the equation $\operatorname{det}\left(B(\lambda) \quad A(\lambda) B(\lambda) \quad \ldots \quad A^{n-1}(\lambda) B(\lambda)\right)=0$, the complementary set is a dense open set of the parameters space.

Example 10: Let us consider the linear system 2 in example 2

This system is clearly controllable because of

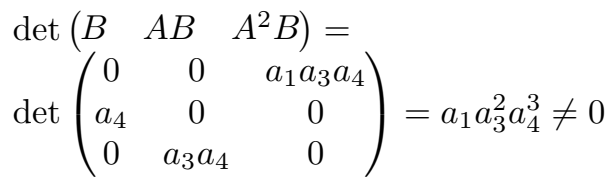

All collection of the same structure systems in a neighborhood $B(\varepsilon)$ with $\varepsilon=\left(\varepsilon_{1}, \varepsilon_{2}, \varepsilon_{3}, \varepsilon_{4}\right),\left|\varepsilon_{i}\right|<\left|a_{i}\right|, i=1,3,4$

$$
\begin{aligned}
& \operatorname{det}\left(\begin{array}{ccc}
0 & 0 & \left(a_{\left.1+\varepsilon_{1}\right)}\left(a_{3}+\varepsilon_{3}\right)\left(a_{4}+\varepsilon_{4}\right)\right. \\
\left(a_{4}+\varepsilon_{4}\right) & 0 & 0 \\
0 & \left(a_{3}+\varepsilon_{3}\right)\left(a_{4}+\varepsilon_{4}\right) & 0
\end{array}\right)= \\
& \left.\left.\left(a_{1}+\varepsilon_{1}\right)\right)\left(a_{3}+\varepsilon_{3}\right)^{2}\left(a_{4}+\varepsilon_{4}\right)\right)^{3} \neq 0
\end{aligned}
$$

Example 11: Let us consider now, the system corresponding to example 3 , in this case if the weights $a_{i j}=1$ for all $i, j$, we have that the system is not controllable because of

$$
\operatorname{rank}\left(\begin{array}{lllll}
B & A B & A^{2} B & \ldots & A^{8} B
\end{array}\right)=6
$$

But considering $a_{i j}=1+\varepsilon_{i j}$ the depending of paramters system is controllable

$$
\operatorname{rank}\left(\begin{array}{lllll}
B & A B & A^{2} B & \ldots & A^{8} B
\end{array}\right)=9
$$

So, the system weights $a_{i j}=1$ for all $i, j$ is structurally stable.

3) Exact controllability: Given a state space representation of a homogeneous linear dynamical system $\dot{x}(t)=A x(t)$ we can ask if it is possible add a control obtaining an equation as in equation 1 , and if it is possible to obtain a good control in other to make the system controllable. That has interest when the solution of the homogeneous system is unstable.

Example 12: Let us consider the homogeneous system corresponding tho following un-weighted graph 6 


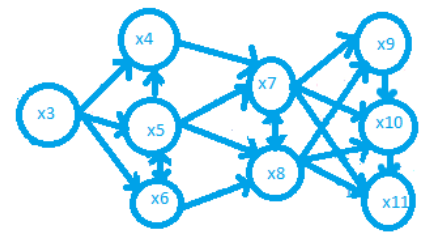

Fig. 6. Un-weighted graph.

The matrix of the system is

$$
A=\left(\begin{array}{lllllllll}
0 & 0 & 0 & 0 & 0 & 0 & 0 & 0 & 0 \\
1 & 0 & 1 & 0 & 0 & 0 & 0 & 0 & 0 \\
1 & 0 & 0 & 1 & 0 & 0 & 0 & 0 & 0 \\
1 & 0 & 1 & 0 & 0 & 0 & 0 & 0 & 0 \\
0 & 1 & 1 & 0 & 0 & 1 & 0 & 0 & 0 \\
0 & 0 & 1 & 1 & 1 & 0 & 0 & 0 & 0 \\
0 & 0 & 0 & 0 & 1 & 1 & 0 & 0 & 0 \\
0 & 0 & 0 & 0 & 1 & 0 & 1 & 0 & 0 \\
0 & 0 & 0 & 0 & 1 & 1 & 0 & 1 & 0
\end{array}\right)
$$

The eigenvalues are: $0,0,0,0,01,1,-1,-1$, so, the system is not stable.

We ask for and external node acting over one or more than one we can obtain a system controllable, and as consequence we can chose stable solutions.

Taking, for example, as $B$, the matrix

$$
B=\left(\begin{array}{ll}
1 & 0 \\
0 & 1 \\
0 & 0 \\
1 & 0 \\
0 & 1 \\
0 & 1 \\
0 & 1 \\
0 & 1 \\
1 & 1
\end{array}\right)
$$

the system $\dot{x}(t)=A x(t)$ is controllable.

It is well known that There are many possible control matrices $B$ in the system 1 that satisfy the controllability condition.

The goal is to find the set of all possible matrices $B$, having the minimum number of columns corresponding to the minimum number $n_{D}(A)$ of independent controllers required to control the whole network.

Definition 13: Let $A$ be a matrix. The exact controllability $n_{D}(A)$ is the minimum of the rank of all possible matrices $B$ making the system 1 controllable.

$$
\begin{aligned}
& n_{D}(A)= \\
& \min \left\{\operatorname{rank} B, \forall B \in M_{n \times i} 1 \leq i \leq n(A, B)\right. \\
& \quad \text { controllable }\} .
\end{aligned}
$$

If confusion is not possible we will write simply $n_{D}$.

It is straightforward that $n_{D}$ is invariant under similarity, that is to say: for any invertible matrix $S$ we have $n_{D}(A)=$ $n_{D}\left(S^{-1} A S\right)$. As a consequence, if necessary we can consider $A$ in its canonical Jordan form.
Example 14: If $A=\operatorname{diag}\left(\lambda_{1}, \ldots, \lambda_{n}\right)$ with $\lambda_{i} \neq \lambda_{j}$ for all $i \neq j$, then $n_{D}=1$, (it suffices to take $B=(1 \ldots 1)^{t}$ ).

Remark 15: Not every matrix $B$ having $n_{D}$ columns is valid to make the system controllable. For example if $A=$ $\operatorname{diag}(1,2,3)$ and $B=(1,0,0)^{t}$, the system $(A, B)$ is not controllable, $\left(\right.$ rank $\left(B \quad A B \quad A^{2} B\right)=1<3$, or equivalently $\operatorname{rank}\left(\begin{array}{ll}A-\lambda I & B\end{array}\right)=2$ for $\lambda=2,3$. Observe that, in this case, the matrix $B$ corresponds to an eigenvector of the operator $A$.

Proposition 16 ( [24]):

$$
n_{D}=\max _{i}\left\{\mu\left(\lambda_{i}\right)\right\}
$$

where $\mu\left(\lambda_{i}\right)=\operatorname{dim} \operatorname{Ker}\left(A-\lambda_{i} I\right)$ is the geometric multiplicity of the eigenvalue $\lambda_{i}$.

Remark 17: Following example 12, computing the ranks

$$
\begin{aligned}
& \operatorname{rank}(A-I)=8 \\
& \operatorname{rank}(A+I)=7 \\
& \operatorname{rank}(A)=7
\end{aligned}
$$

we deduce that minimum number of columns in matrix $B$ making the system controllable is two.

\section{Controllability of MUltiagent NeURAL NETWORKS}

The complexity of the brain drives that in order to study control problems, the global model is divided into several local submodels, each with its complex and interrelated network structure. Structuring, in this way, the brain as a neuronal multi-network with a common goal.

Let us consider a group of $k$ agents. The dynamic of each agent is given by the following linear dynamical systems

$$
\begin{aligned}
\dot{x}^{1}(t) & =A_{1} x^{1}(t)+B_{1} u^{1}(t) \\
\vdots & \\
\dot{x}^{k}(t) & =A_{k} x^{k}(t)+B_{k} u^{k}(t)
\end{aligned}
$$

$A_{i} \in M_{n}(\mathbb{R}), B_{i} \in M_{n \times m}(\mathbb{R}), x^{i}(t) \in \mathbb{R}^{n}, u^{i}(t) \in \mathbb{R}^{m}$, $1 \leq i \leq k$.

We consider the undirected graph $\mathcal{G}$ with

i) Vertex set: $V=\{1, \ldots, k\}$

ii) Edge set: $\mathcal{E}=\{(i, j) \mid i, j \in V\} \subset V \times V$

defining the communication topology among agents.

Writing

$$
\begin{gathered}
\mathcal{X}(t)=\left(\begin{array}{c}
x^{1}(t) \\
\vdots \\
x^{k}(t)
\end{array}\right), \quad \dot{\mathcal{X}}(t)=\left(\begin{array}{c}
\dot{x}^{1}(t) \\
\vdots \\
\dot{x}^{k}(t)
\end{array}\right), \\
\mathcal{U}(t)=\left(\begin{array}{c}
u^{1}(t) \\
\vdots \\
u^{k}(t)
\end{array}\right), \\
\mathcal{A}=\left(\begin{array}{ccc}
A_{1} & \\
& \ddots & \\
& & A_{k}
\end{array}\right), \mathcal{B}=\left(\begin{array}{ccc}
B_{1} & \\
& \ddots & \\
& & B_{k}
\end{array}\right),
\end{gathered}
$$

Following this notation we can describe the multisystem as a system: 


$$
\dot{\mathcal{X}}(t)=\mathcal{A X}(t)+\mathcal{B U}(t)
$$

and we are interested in take the output of the system to a reference value and keep it there, we can ensure that if the system is controllable. Clearly, this system is controllable if and only if each subsystem is controllable, and, in this case, there exist a feedback in which we obtain the desired solution.

But, in our case, not all possible feedbacks are available due the restriction of interconnection of agents. So we are interested in a feedbacks $K_{i}$ in such a way that the with control

$$
u^{i}(t)=K_{i} \sum_{j \in \mathcal{N}_{i}}\left(x^{i}(t)-x^{j}(t)\right), 1 \leq i \leq k
$$

the system has prescribed eigenvalues in order to take a desired output of the system.

In our particular setup, we are interested in a solution such that

$$
\lim _{t \rightarrow \infty}\left\|x^{i}-x^{j}\right\|=0,1 \leq i, j \leq k .
$$

That is to say, founding solutions of each subsystem arriving all, to the same point.

Proposition 18: Taking the control $u^{i}(t)=$ $K \sum_{j \in \mathcal{N}_{i}}\left(x^{i}(t)-x^{j}(t)\right), \quad 1 \leq i \leq k$ the closed-loop system can be described as

$$
\dot{\mathcal{X}}(t)=\left(\mathcal{A}+\mathcal{B K}\left(\mathcal{L} \otimes I_{n}\right)\right) \mathcal{X}(t) .
$$

where $\mathcal{K}=\left(\begin{array}{lll}K_{1} & & \\ & \ddots & \\ & & K_{k}\end{array}\right)$.

Computing the matrix $\mathcal{A}+\mathcal{B K}\left(\mathcal{L} \otimes I_{n}\right)$ we obtain

$$
\left(\begin{array}{cccc}
A_{1}+l_{11} B_{1} K_{1} & l_{12} B_{1} K_{1} & \ldots & l_{1 k} B_{1} K_{1} \\
l_{21} B_{2} K_{2} & A_{2}+l_{22} B_{2} K_{2} & \ldots & l_{2 k} B_{2} K_{2} \\
\vdots & \vdots & \ddots & \vdots \\
l_{k 1} B_{k} K_{k} & l_{k 2} B_{k} K_{k} & \ldots & A_{k}+l_{k k} B_{k} K_{k}
\end{array}\right)
$$

Example 19: We consider 3 agents with the following dynamics of each agent

$$
\begin{aligned}
& \dot{x}^{1}=A_{1} x^{1}+B_{1} u^{1} \\
& \dot{x}^{2}=A_{2} x^{2}+B_{2} u^{2} \\
& \dot{x}^{3}=A_{3} x^{3}+B_{3} u^{3}
\end{aligned}
$$

with $A_{1}=A_{2}=A_{3}=\left(\begin{array}{cc}0 & 1 \\ -0.1 & -0.5\end{array}\right)$, and $B_{1}=B_{2}=$ $B_{3}=\left(\begin{array}{l}0 \\ 1\end{array}\right)$.

The communication topology is defined by the graph $(\mathcal{V}, \mathcal{E})$ : $V=\{1,2,3\}$

$\mathcal{E}=\{(i, j) \mid i, j \in V\}=\{(1,2),(1,3),(2,1),(3,1)\} \subset$ $V \times V$

The neighbors of the parent nodes are $\mathcal{N}_{1}=\{2,3\}, \mathcal{N}_{2}=$ $\{1\}, \mathcal{N}_{3}=\{1\}$.

The Laplacian matrix of the graph is

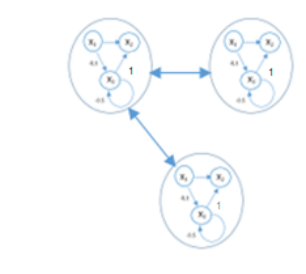

Fig. 7. Multiagent graph

$$
\mathcal{L}=\left(\begin{array}{ccc}
2 & -1 & -1 \\
-1 & 1 & 0 \\
-1 & 0 & 1
\end{array}\right)
$$

Taking $K_{i}=K=\left(\begin{array}{ll}k & \ell\end{array}\right)$, for $i=1,2,3$

The matrix $\mathcal{M}$ of the system is

$$
\left(\begin{array}{cccccc}
0 & 1 & 0 & 0 & 0 & 0 \\
2 k-\frac{1}{10} & 2 \ell-\frac{1}{2} & -k & -\ell & -k & -\ell \\
0 & 0 & 0 & 1 & 0 & 0 \\
-k & -\ell & k-\frac{1}{10} & \ell-\frac{1}{2} & 0 & 0 \\
0 & 0 & 0 & 0 & 0 & 1 \\
-k & -\ell & 0 & 0 & k-\frac{1}{10} & \ell-\frac{1}{2}
\end{array}\right)
$$

Taking $K=\left(\begin{array}{ll}-0.5 & -0.2\end{array}\right)$ the eigenvalues are $-0.5500+$ $1.1391 i,-0.5500-1.1391 i,-0.2500+0.1936 i,-0.2500-$ $0.1936 i,-0.3500+0.6910 i,-0.3500-0.6910 i$, then the system has a stable solution and the the three trajectories arrive at a common point.

As we can see in the example, all agents on the multi-agent system, have an identical linear dynamic mode, and in this case, we can consider the same feedback for each system.

In this particular case proposition 18 can be rewritten in the following manner (see [4], [22])

Proposition 20: Taking the control $u^{i}(t)=$ $K \sum_{j \in \mathcal{N}_{i}}\left(x^{i}(t)-x^{j}(t)\right), 1 \leq i \leq k$ the closed-loop system for a multiagents having identical linear dynamical mode, can be described as

$$
\dot{\mathcal{X}}=\left(\left(I_{k} \otimes A\right)+\left(I_{k} \otimes B K\right)\left(\mathcal{L} \otimes I_{n}\right)\right) \mathcal{X} .
$$

1) Structural Controllability of Multi-Agent Systems with Fixed Topology: Let us consider a group of $k$ agents. The dynamic of each agent is given by the linear dynamical systems as 5 with external control inputs, that is to say

$$
\begin{aligned}
\dot{x}^{1}(t) & =A_{1} x^{1}(t)+B_{1} u^{1}(t)+D_{i} u_{\mathrm{ext}}^{1}(t) \\
\vdots & \\
\dot{x}^{k}(t) & =A_{k} x^{k}(t)+B_{k} u^{k}(t)+D_{k} u_{\mathrm{ext}}^{k}(t)
\end{aligned}
$$

$A_{i} \in M_{n}(\mathbb{R}), B_{i} \in M_{n \times m}(\mathbb{R}), D_{i} \in M_{n \times p}(\mathbb{R}), x^{i}(t) \in \mathbb{R}^{n}$, $u^{i}(t) \in \mathbb{R}^{m}, u_{\text {ext }}^{i}(t) \in \mathbb{R}^{p}$ the external control input of the agent $i, 1 \leq i \leq k$.

Given the following protocol as 6 where $K$ is the feedback gain matrix, and defining

$$
\mathcal{U}_{\text {ext }}(t)=\left(\begin{array}{c}
u_{\text {ext }}^{1}(t) \\
\vdots \\
u_{\text {ext }}^{k}(t)
\end{array}\right), \mathcal{D}=\left(\begin{array}{ccc}
D_{1} & & \\
& \ddots & \\
& & D_{k}
\end{array}\right)
$$


Proposition 21: With these notations the system can be described as

$$
\dot{\mathcal{X}}(t)=\left(\mathcal{A}+\mathcal{B K}\left(\mathcal{L} \otimes I_{n}\right)\right) \mathcal{X}(t)+\mathcal{D} \mathcal{U}_{\text {ext }}(t) .
$$

In the particular case where all agents on the multi-agent system, have an identical linear dynamic mode, we have the following corollary

Corollary 22: For the case where all agents on the multiagent system, have an identical linear dynamic mode, the system can be described as

$$
\dot{\mathcal{X}}=\left(\left(I_{k} \otimes A\right)+\left(I_{k} \otimes B K\right)\left(\mathcal{L} \otimes I_{n}\right)\right) \mathcal{X}+\mathcal{D} \mathcal{U}_{\text {ext }}(t) .
$$

The expression of the multi-agent system as a linear system permit us to adapt the structural controllability concept to the multi-agent system.

Definition 23: The multi-agent system 9 is said to be structurally controllable if one can change the non-zero entries of $\left.\left(\mathcal{A}+\mathcal{B K}\left(\mathcal{L} \otimes I_{n}\right)\right), \mathcal{D}\right)$ for some particular values from $\mathbb{R}$ such that system 9 is controllable in the classical sense.

Example 24: Let us consider the example 19 with external input matrix $D=\left(\begin{array}{l}1 \\ 0\end{array}\right)$ and $k=0.1$ and $l=0.2$

The system is not controllable because of $\operatorname{rank}\left(\mathcal{D} \quad \mathcal{M D} \quad \ldots \quad \mathcal{M}^{5} \mathcal{D}\right)=5<6$.

Changing

$$
\mathcal{M}=\left(\begin{array}{cccccc}
0 & 1 & 0 & 0 & 0 & 0 \\
0.1 & -0.1 & -0.1 & -0.2 & -0.1 & -0.2 \\
0 & 0 & 0 & 1 & 0 & 0 \\
-0.1 & -0.2 & 0 & -0.3 & 0 & 0 \\
0 & 0 & 0 & 0 & 0 & 1 \\
-0.1 & -0.2 & 0 & 0 & 0 & -0.3
\end{array}\right)
$$

by

$$
\overline{\mathcal{M}}=\left(\begin{array}{cccccc}
0 & 1 & 0 & 0 & 0 & 0 \\
-0.1 & -0.2 & 0.1 & -0.2 & -0.1 & -0.2 \\
0 & 0 & 0 & 1 & 0 & 0 \\
-0.1 & -0.2 & 0 & 0.3 & 0 & 0 \\
0 & 0 & 0 & 0 & 0 & 1 \\
-0.1 & -0.2 & 0 & 0 & 0 & -0.3
\end{array}\right)
$$

the new system is controllable, because of $\operatorname{rank}\left(\mathcal{D} \quad \overline{\mathcal{M}} \mathcal{D} \quad \ldots \quad \overline{\mathcal{M}}^{5} \mathcal{D}\right)=6$.

2) Exact controllability: Pollowing [6], let us consider a group of $k$ agents. The dynamic of each agent is given by the following homogeneous linear dynamical systems

$$
\begin{aligned}
\dot{x}^{1}(t) & =A_{1} x^{1}(t) \\
\vdots & \\
\dot{x}^{k}(t) & =A_{k} x^{k}(t)
\end{aligned}
$$

$x^{i}(t) \in \mathbb{R}^{n}, 1 \leq i \leq k$.

We ask for minimum number of columns that a matrix $B$ must have for the system

$$
\begin{aligned}
\dot{x}^{1}(t) & =A_{1} x^{1}(t)+B u^{1}(t) \\
\vdots & \\
\dot{x}^{k}(t) & =A_{k} x^{k}(t)+B u^{k}(t)
\end{aligned}
$$

to be controllable (the matrix $B$ the same for each agent).

Let $\lambda_{i_{1}}, \ldots, \lambda_{i_{r_{i}}}$ the eigenvalues of the matrix $A_{i}$ with geometrical multiplicities $\mu\left(\lambda_{i_{1}}\right), \ldots, \mu\left(\lambda_{i_{r_{i}}}\right)$. Then

Proposition 25:

$$
\begin{aligned}
& n_{D}(\mathcal{A})= \\
& \max \left(\mu\left(\lambda_{1_{1}}\right), \ldots, \mu\left(\lambda_{1_{r_{1}}}\right), \ldots, \mu\left(\lambda_{k_{1}}\right), \ldots, \mu\left(\lambda_{k_{r_{k}}}\right)\right.
\end{aligned}
$$

Corollary 26:

$$
n_{D}(\mathcal{A})=n_{D}\left(A_{i}\right)
$$

for some $i=1, \ldots, k$.

Remark 27:

- Not all matrices $B$ having $n_{D}\left(A_{i}\right)$ columns and making the system $\dot{x}^{i}=A_{i} x^{i}+B u^{i}$ controllable are available for the multiagent system.

- Taking all possible matrices $B$ making the system controllable we can consider the existence of the matrix $K$. Example 28: Suppose $A_{1}=\left(\begin{array}{ll}2 & \\ & 3\end{array}\right), A_{2}=\left(\begin{array}{ll} & 1 \\ 1 & \end{array}\right)$.

The matrix $B=\left(\begin{array}{l}1 \\ 1\end{array}\right)$ make the system $\dot{x}(t)=A_{1} x(t)+$ $B u(t)$ controllable but not $\dot{x}(t)=A_{2} x(t)+B u(t)$.

The matrix $B=\left(\begin{array}{l}1 \\ 0\end{array}\right)$ make the system $\dot{x}(t)=A_{2} x(t)+$ $B u(t)$ controllable but not $\dot{x}(t)=A_{1} x(t)+B u(t)$.

Nevertheless, the matrix $B=\left(\begin{array}{l}1 \\ 2\end{array}\right)$ make both systems controllable.

Introducing topology relating the system, the exact controllability is formulated as follows.

Definition 29: The system 5 with topology 6 is exact controllable, if there exist external inputs $D$, making the system 8 controllable.

Example 30: The system given in example 17 withe the same values for $k$ and $\ell$ is exact controllable, it suffices to consider $D=\left(\begin{array}{l}0 \\ 1\end{array}\right)$.

\section{CONCLUSIONS}

Different aspects of controllability for multi-agent systems with a topology connecting the agents conforming to the multisystem and where all agents have an identical linear dynamic mode are analyzed, concretively aspects about structural controllability and exact controllability. Structural controllability theory offers a general tool to control structured linear systems, in this paper, it is proved that this tool can be generalized to multi-systems in which each of the systems has a predetermined structure. In systems theory there are results on the minimum number of controls necessary to make the system controllable. Important challenges arise when trying to determine this number for multisystems, here we have made a contribution in this regard.

\section{REFERENCES}

[1] J. Assan, A. Lafay, A. Perdon, "Computation of maximal precontrollability submodules over a Noetherian ring" Systems \& Control Letters, vol. 2(3), pp. 153-161, 1999.

[2] F. Cardetti, M. Gordina, "A note on local controllability on lie groups". Systems \& Control Letters, vol. 57, pp. 978-979, 2008. 
[3] C.T. Chen, Introduction to Linear System Theory. Holt, Rinehart and Winston Inc, New York, 1970.

[4] M.I. Garcia-Planas. "Obtaining Consensus of Multi-agent Linear Dynamic Systems". Advances in Applied and Pure Mathematics, pp.1-5, 2005.

[5] M.I. García-Planas, "Generalized Controllability Subspcaes for Timeinvariant Singular Linear Systems", Proceedings of Physcon 2011, IPACS Electronic Library, 2011.

[6] M.I. García-Planas, "Analyzing Controllability of Neural Networks", Wseas Transactions on Circuits and Systems, vol. 18, pp. 1-6, 2019.

[7] M.I. García-Planas, "Exact Controllability of Linear Dynamical Systems: a Geometrical Approach", Applications of Mathematics, vol. 1, pp. 37-47, 2017, DOI:10.21136/AM.2016.0427-15

[8] M.I. Garcia-Planas, J.L. Domínguez, "Alternative tests for functional and pointwise output-controllability of linear time-invariant systems", Systems \& control letters, vol. 62(5), pp. 382-387, 2013.

[9] M. I. García-Planas, M.V. García-Camba Vives, M. V. Dyscalculia, mind, calculating brain and education. In EDULEARN18: 10th Annual International Conference on Education and New Learning Technologies: Palma de Mallorca, Spain: July 2-4, 2018: proceedings book, (2018). pp. 0480-0489.

[10] Sh, Gu, F. Pasqualetti, M. Cieslak, Q. K. Telesford, A. B. Yu, A. E. Kahn, J. D. Medaglia, J. M. Vettel, M. B. Miller, S. T. Grafton, D. S. Bassett. "Controllability of structural brain networks"2015. DOI: $10.1038 /$ ncomms 9414

[11] M. L. J. Hautus, "Controllability and observability conditions of linear autonomous systems". Nederl. Akad. Wetensch. Proc. Ser. A $72=$ Indag. Math., vol. 31, pp. 443-448, 1969.

[12] A. Heniche, I. Kamwa, "Using measures of controllability and observability for input and output selection", Proceedings of the 2002 IEEE International Conference on Control Applications, vol. 2, pp. 12481251, (2002)

[13] R. E. Kalman, P. L. Falb and M. A. Arbib, "Topics in Mathematical Control Theory", McGraw-Hill Book Co., New York-Toronto, Ont.London 1969.

[14] N. Kriegeskorte Deep Neural Networks: "A New Framework for Modeling Biological Vision and Brain Information Processing". Annual Review of Vision Science, vol. 1, pp. 417-46, 2015.

[15] P. Kundur, Power System Stability and Control, McGraw-Hill, 1994.

[16] P. Lancaster, M. Tismenetsky, "The Thoery of Matrices". Academic Press. San Diego (1985).

[17] D. Leitold, A. Vathy-Fogarassy, J. Abonyi. "Controllability and observability in complex networks - the effect of connection types". Scientific Reports, vol. 7(151), 2017. pmid:28273948

[18] C. Lin, Structural controllability, IEEE Trans. Automat. Contr., vol. 19, pp. 201-208, 1974.

[19] Y. Liu, J. Slotine, A. Barabási, "Controllability of complex networks ", Nature, vol. 473(7346), pp. 167-173, 2011.

[20] R. Shields, J. Pearson, "Structural controllability of multi-input linear systems". IEEE Trans. Automat. Contr, vol. 21, pp. 203-212, 1976.

[21] O. Sporns, "Graph theory methods: applications in brain networks". Dialogues in Clinical Neuroscience, vol. 20(2), pp. 111-120, 2018.

[22] J. Wang, D. Cheng, X. Hu, "Consensus of multi-agent linear dynamics systems"Asian Journal of Control, vol. 10(2), pp. 144-155, 2008.

[23] D. West Introduction to Graph Theory. Prentice Hall (3rd Edition), 2007.

[24] Z.Z. Yuan, C. Zhao, W.X. Wang, Z.R. Di, Y.C. Lai, "Exact controllability of multiplex networks", New Journal of Physics, vol. 16, pp. 1-24, 2014. 